\title{
On the dependence structure between S\&P500, VIX and implicit Interexpectile Differences
}

\author{
Fabio Bellini†, Lorenzo Mercurił and Edit Rroji \\ $\dagger$ Department of Statistics and Quantitative Methods, \\ University of Milano-Bicocca, \\ $\ddagger$ Department of Economics, Management and Quantitative Methods, \\ University of Milan \\ and CREST Japan Science and Technology Agency, Tokyo \\ ${ }^{\diamond}$ Department of Mathematics, \\ Politecnico di Milano.
}

\begin{abstract}
We study the dependence structure between the S\&P500, the VIX Index, and implicit Interexpectile Differences, that are an alternative measure of implied volatility based on the notion of implicit expectile, recently introduced in Bellini et al. (2018a). After filtering the time series of the marginals by ARMA(E)GARCH models, we fit several parametric families of copulas to the pairwise joint distribution of the residuals, in order to investigate the presence of radial asymmetry and asymptotic tail dependence.
\end{abstract}

Keywords: VIX, Implicit Expectiles, Interexpectile Differences, ARMA-GARCH, Copulas.

JEL Classification: C00, C02, C53, G31

Extracting information about the risk neutral distribution of a financial index from the prices of options written on it is a central issue in financial econometrics. As far as the variability of the risk neutral distribution is concerned, the standard approach is to measure it by means of the model-free implied volatility, computed by the method suggested first in Demeterfi et al. (1999) and later implemented by the CBOE (2009) in the computation of the VIX Index, that represents the 30-days implied volatility of the S\&P500 Index.

The VIX index is nowadays extremely popular among practitioners and is itself the underlying of a number of quoted derivatives such as future contracts and plain vanilla call and put options. It is often believed that the VIX index may carry some useful information on the expectation of future logreturns or on the future realized variance; this topic has been investigated in details in the literature on the econometrics of option pricing (see e.g. Banerjee et al. (2007), Rubbaniy et al. (2014), and the references therein).

It is also a generally accepted stylized fact the negative dependence between contemporaneous variations of the S\&P500 Index and variations of the VIX Index, a phenomenon called leverage effect, that has been investigated by means of copula functions in several papers.

In Ammann and Süss (2009) the authors considered several implied volatility indices and fitted a skew-t copula on the residuals of autoregressive $\operatorname{GARCH}(1,1)$ models with skew-t innovations. It is indeed generally believed that a necessary first step of the analysis is to eliminate serial

Corresponding author. Email: edit.rroji@polimi.it 
autocorrelations of the marginals, otherwise the resulting copula model might be misspecified. The main conclusion of Ammann and Süss was that an asymmetric negative dependence exists, and becomes more pronounced during periods of financial turmoils. On the contrary, in Peng and $\mathrm{Ng}$ $(2012 \mathrm{a}, \mathrm{b})$ the authors adopted a dynamic copula approach following Patton (2006), among others. After fitting ARMA-GARCH models to the marginals, the authors fitted on the residuals a timevarying symmetrized Joe-Clayton copula, with parameters depending on time in an autoregressive fashion. Finally, in Fink et al. (2017) regime-switching vine copula models have been applied to the multivariate distribution of equity and implied volatility indices, in order to analyze contagion and spillover effect.

Despite its popularity, the VIX Index is not the only methodology available for measuring the variability of the risk neutral distribution. An alternative approach has been suggested in Bellini et al. (2018a), where the notions of implicit expectiles and implicit Interexpectiles Differences have been introduced. The main idea is that from the prices of european calls and puts it is very easy to determine the expectiles of the risk neutral distribution (see Section 1), in principle in modelfree way and without any discretization or truncation error. The difference between two implicit expectiles corresponding to different thresholds, similarly to the interquantile difference widely used in exploratory data analysis, can then serve as a natural measure of the variability of the risk neutral distribution, with the advantage of being consistent with respect to the convex order.

In Bellini et al. (2018a) it was shown, although on a relatively limited dataset of option prices on the italian stock index FTSEMIB in the years 2008-2009, that implicit Interexpectile Differences are strongly correlated with the Italian VIX and indeed may constitute interesting alternative implied variability measures, carrying also some forecasting power on future logreturns.

We present an investigation of the dependence between the S\&P 500, the VIX index and Interexpectile Differences. The dataset is composed of european call and put options written on the S\&P 500 for the time period 2003-2013. The paper is structured as follows. In Section 1 we recall the definition of Expectiles and implicit Interexpectile Differences and compare them with the VIX from a theoretical point of view. In Section 2 we set the basic notations for ARMA-(E)GARCH models and present the parametric families of copulas that will be used in the empirical analysis of Section 3. Section 4 concludes.

\section{Measures of implied variability}

In this section we review the definition and the computation of two measures that capture the implied volatility in the market. The first is the VIX index that has been extensively used to measure the perceived market risk while the second measure called Interexpectile Difference has been recently proposed in Bellini et al. (2018a). The VIX index is designed to represent a 30-day implied volatility and is computed as the square root of a time-weighted average of the implied variances corresponding to the two maturities closer to 30 days. Each implied variance is computed by the well-known formula

$$
\sigma_{T}^{2}=\frac{2}{T} \sum_{i} \frac{\Delta K_{i}}{K_{i}^{2}} e^{r T} O\left(K_{i}\right)-\frac{1}{T}\left(\frac{F}{K_{0}}-1\right)^{2},
$$

where $O\left(K_{i}\right)$ is the price of an out of the money call or put with strike $K_{i}, \Delta K_{i}$ is the difference between two consecutive strikes, $F$ is the forward price and $K_{0}$ is the highest quoted strike lower than F. As shown by Demeterfi et al. (1999), this quantity is a discretization of the risk neutral expectation of the realized variance, that is an idealized situation of a continuum of strikes can be written also as

$$
\sigma_{T}^{2}=\frac{2 e^{r T}}{T}\left(\int_{0}^{F} \frac{P(K)}{K^{2}} d K+\int_{F}^{+\infty} \frac{C(K)}{K^{2}} d K\right)
$$


$r$ is the risk-free rate, $T$ is the maturity.

The VIX is thus equal to the standard deviation of the risk neutral distribution, and is computed by means of all out of the money available call and put prices; it is a pure measure of variability. The expectiles $e_{\tau}$ have been introduced in Newey and Powell (1987) for $X \in L^{2}(\Omega, \mathcal{F}, \mathbb{P})$ as follows:

$$
e_{\tau}(X):=\underset{x \in \mathbb{R}}{\operatorname{argmin}} \mathbb{E}\left[\tau(X-x)_{+}^{2}+(1-\tau)(X-x)_{-}^{2}\right], \quad \tau \in(0,1) .
$$

Observe that $e_{1 / 2}(X)=\mathbb{E}[X]$, so the expectiles are a one-parameter family that includes the mean as a special case. Equivalently, for any $X \in L^{1}(\Omega, \mathcal{F}, \mathbb{P})$, the expectile $e_{\tau}(X)$ can be defined as the unique solution of the equation

$$
\tau \mathbb{E}\left[\left(X-e_{\tau}(X)\right)_{+}\right]=(1-\tau) \mathbb{E}\left[\left(X-e_{\tau}(X)\right)_{-}\right] .
$$

Expectiles are an asymmetric generalization of the mean (see e.g. Bellini et al. 2018b) and the expectile curve $\tau \mapsto e_{\tau}$ completely determines a distribution. Moreover, from Bellini et al. (2014) expectiles with $\tau \geq 1 / 2$ are the only generalized quantiles that are coherent risk measures.

In order to compute implicit expectiles, we recall that

$$
C(K)=e^{-r T} \mathbb{E}_{\mathbb{Q}}\left[\left(S_{T}-K\right)_{+}\right], \quad P(K)=e^{-r T} \mathbb{E}_{\mathbb{Q}}\left[\left(S_{T}-K\right)_{-}\right]
$$

where $S_{T}$ is the price of the underlying at maturity and $\mathbb{Q}$ is the risk neutral measure. From (2) it follows that the implicit $\tau$-expectile is the unique strike price $\bar{K}$ such that

$$
\tau C(\bar{K})=(1-\tau) P(\bar{K})
$$

The case $\tau=1 / 2$ gives the forward price that is indeed the risk neutral expectation of the underlying at maturity.

The Interexpectile Difference $\Delta_{\tau}$ is defined as follows:

$$
\Delta_{\tau}=e_{\tau}(X)-e_{1-\tau}(X),
$$

for $X \in L^{1}$ and $\tau \in(1 / 2,1)$ (see Fig. 1). As shown in Bellini et al. (2018a), it satisfies several desirable properties that we recall in the following.

Theorem 1.1 Let $X \in L^{1}, \tau \in(1 / 2,1)$ and $\Delta_{\tau}$ as in (1). Then:

a) $\Delta_{\tau}(X) \geq 0$ and $\Delta_{\tau}(X)=0$ if and only if $X=c \mathbb{P}$-a.s.

b) $\Delta_{\tau}(X)=\Delta_{\tau}(-X)$

c) $\Delta_{\tau}(X)$ is strictly increasing and continuous in $\tau$

d) $\Delta_{\tau}(X) \rightarrow 0^{+}$for $\tau \rightarrow 1 / 2^{+}$and $\Delta_{\tau}(X) \rightarrow \operatorname{esssup}(X)-\operatorname{essinf}(X)$ for $\tau \rightarrow 1^{-}$

e) $\Delta_{\tau}(X+h)=\Delta_{\tau}(X)$, for $h \in \mathbb{R}$

f) $\Delta_{\tau}(\lambda X)=\lambda \Delta_{\tau}(X)$, for $\lambda \geq 0$

g) $\Delta_{\tau}(X+Y) \leq \Delta_{\tau}(X)+\Delta_{\tau}(Y)$

h) $X \leq_{c x} Y \Rightarrow \Delta_{\tau}(X) \leq \Delta_{\tau}(Y)$,

where $\leq_{c x}$ denotes the usual convex order.

The Interexpectile Difference is a natural analog to the interquantile difference, that is widely used in exploratory data analysis, but has the advantage of being a true measure of variability, since it is consistent with the convex order. 


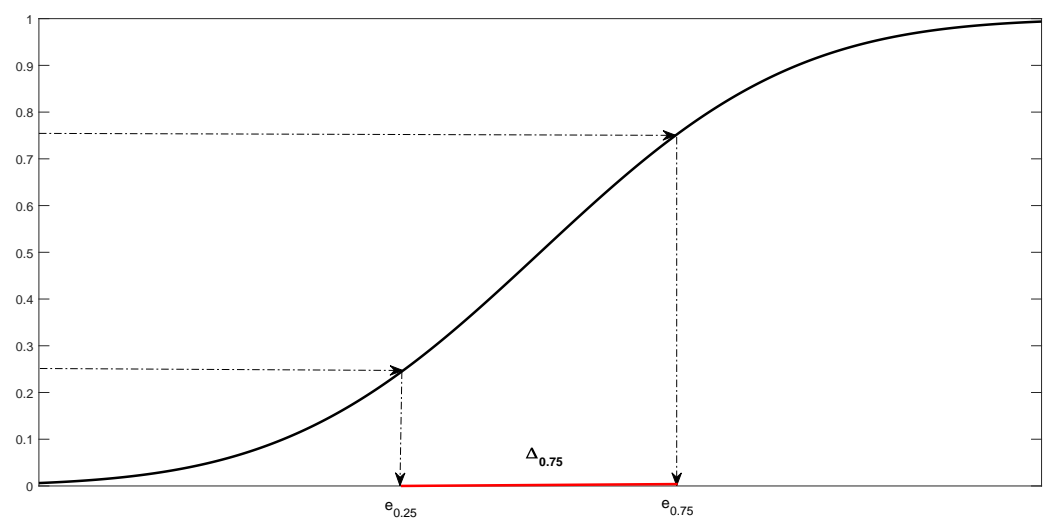

Figure 1. Graphical illustration of the Interexpectile Difference $\Delta_{0.75}$.

\section{Copula-(E)GARCH models}

We start by briefly recalling the basic definitions and notations on bivariate copulas, referring to the monographs Nelsen (2006), Joe (2014), Mai and Scherer (2014) for further details. A bivariate copula $C:[0,1] \times[0,1] \rightarrow[0,1]$ is the distribution function of a two-dimensional random vector with uniform marginals. According to Sklar's theorem, given a random vector $(X, Y)$ with joint distribution function $F(x, y)=\mathbb{P}(X \leq x, Y \leq y)$ and with continuous marginals' distributions $F_{X}=\mathbb{P}(X \leq x)$ and $F_{Y}=\mathbb{P}(Y \leq y)$, there exists a unique copula $C$ such that

$$
F(x, y)=C\left(F_{X}(x), F_{Y}(y)\right) .
$$

A copula is said to be exchangeable if $C\left(u_{2}, u_{1}\right)=C\left(u_{1}, u_{2}\right)$, and radially symmetric if $C=\bar{C}$, where the so called survival copula $\bar{C}$ is defined as

$$
\bar{C}(u, v)=u+v-1+C(1-u, 1-v) .
$$

From a graphical point of view, exchangeability corresponds to symmetry with respect to the SWNE diagonal of the unit square, while radial symmetry corresponds to symmetry with respect to the NW-SE diagonal. A standard concordance measure for a random vector $(X, Y)$ is Kendall's $\tau$,

$$
\tau(X, Y):=\int_{0}^{1} \int_{0}^{1} C(u, v) d C(u, v)-1
$$

where $C$ is the copula of $(X, Y)$. As it is well known, Kendall's $\tau$ can be interpreted as the difference between the probability of concordance and the probability of discordance in two independent copies $\left(X_{1}, Y_{1}\right),\left(X_{2}, Y_{2}\right)$ of the random vector $(X, Y)$, as follows:

$$
\tau(X, Y)=\mathbb{P}\left(\left(X_{1}-X_{2}\right)\left(Y_{1}-Y_{2}\right)>0\right)-\mathbb{P}\left(\left(X_{1}-X_{2}\right)\left(Y_{1}-Y_{2}\right)<0\right) .
$$

For a given copula $C(u, v)$, its coefficients of lower and upper tail dependence are defined by

$$
\lambda_{L}=\lim _{u \rightarrow 0^{+}} \frac{C(u, u)}{u}, \quad \lambda_{U}=\lim _{u \rightarrow 0^{+}} \frac{\bar{C}(u, u)}{u} .
$$

If there exist two functions $\ell_{1}$ and $\ell_{2}$ slowly varying at $0^{+}$such that for $u \rightarrow 0^{+}$

$$
C(u, u) \sim u^{\kappa_{L}} \ell_{1}(u), \quad \bar{C}(u, u) \sim u^{\kappa_{U}} \ell_{2}(u),
$$


then $\kappa_{L}$ and $\kappa_{U}$ are called respectively the lower and the upper tail order of $C$. Letting $\kappa=$ $\kappa_{L}$ or $\kappa_{U}$, a distinction can be made (see e.g. Joe (2014)) between strong tail dependence (if $\kappa=1$ ), intermediate tail dependence (if $1<\kappa<2$ ) and asymptotic tail independence (if $\kappa=2$ ). Clearly, if $\kappa>1$ the corresponding tail dependence coefficient $\lambda$ is equal to 0 . In the paper we will consider mainly the following one-parameter and two-parameter families of exchangeable copulas:

$$
\begin{aligned}
& C_{\text {Frank }}(u, v ; \delta)=-\frac{1}{\delta} \ln \left(\frac{1-e^{-\delta}-\left(1-e^{-\delta u}\right)\left(1-e^{-\delta u}\right)}{1-e^{-\delta}}\right), \quad \delta \in \mathbb{R} \\
& C_{\text {Clayton }}(u, v ; \theta)=\left(u^{-\theta}+v^{-\theta}-1\right)^{-\frac{1}{\theta}}, \quad \theta>0 \\
& C_{\text {Gumbel }}(u, v ; \theta)=\exp \left(-\left[(-\ln u)^{\theta}+(-\ln v)^{\theta}\right]^{1 / \theta}\right), \quad \theta \geq 1 \\
& C_{B B 1}(u, v ; \theta, \delta)=\left\{1+\left[\left(u^{-\theta}-1\right)^{\delta}+\left(v^{-\theta}-1\right)^{\delta}\right]^{1 / \delta}\right\}^{-1 / \theta}, \quad \theta>0, \delta \geq 1
\end{aligned}
$$

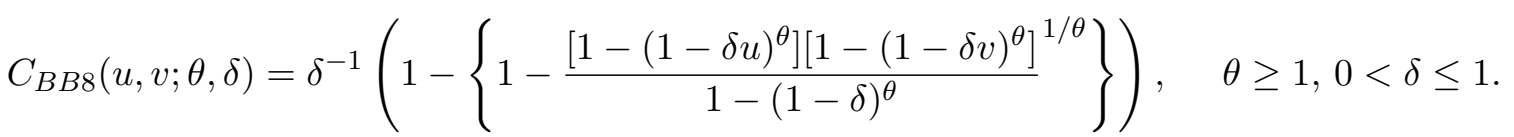

The Frank copula is the only radially symmetric one and has asymptotic upper and lower tail independence $\left(\kappa_{L}=\kappa_{U}=2\right)$. The Clayton copula has upper asymptotic tail independence and lower strong tail dependence with $\lambda_{L}=2^{-1 / \theta}$. The Gumbel copula has intermediate lower tail dependence with $\kappa_{L}=2^{1 / \theta}$ and upper strong tail dependence with $\lambda_{U}=2-2^{1 / \theta}$. The BB1 copula is a two-parameter family admitting the Clayton copula as a special case for $\delta=1$ and the Gumbel copula as a limiting case for $\theta \rightarrow 0^{+}$. It has upper and lower strong tail dependence with $\lambda_{L}=2^{-1 /(\delta \theta)}$ and $\lambda_{U}=2-2^{1 / \delta}$. The BB8 copula has lower and upper asymptotic tail independence and admits the Frank copula as a limit for $\theta \rightarrow+\infty$. In order to be able to model negative dependence, for a given copula $C(u, v)$ we consider also the "rotated" versions

$$
\begin{aligned}
C^{90}(u, v) & =v-C(1-u, v) \\
C^{270}(u, v) & =u-C(u, 1-v),
\end{aligned}
$$

that for exchangeable copulas correspond to counterclockwise rotations of $90^{\circ}$ and $270^{\circ}$ degrees. In order to remove serial autocorrelation in the time series of S\&P500, VIX and Interexpectile Differences, we fit ARMA models of the form

$$
r_{t}=\mu+\sum_{i=1}^{r} \phi_{i}\left(r_{t-i}-\mu\right)+\sum_{i=1}^{s} \varphi_{i} \epsilon_{t-i}+\epsilon_{t}
$$

where $\epsilon_{t}=\sigma_{t} z_{t}$, with $z_{t}$ i.i.d. For the conditional variance $\sigma_{t}^{2}$ we consider GARCH and EGARCH specifications given respectively by

$$
\sigma_{t}^{2}=\omega+\sum_{i=1}^{p} \alpha_{i} \epsilon_{t-i}^{2}+\sum_{i=1}^{q} \beta_{i} \sigma_{t-i}^{2}
$$

and

$$
\ln \left(\sigma_{t}^{2}\right)=\omega+\sum_{i=1}^{p}\left[\alpha_{i} z_{t-i}+\gamma_{i}\left(\left|z_{t-i}\right|-\mathbb{E}\left(z_{t-i}\right)\right)\right]+\sum_{i=1}^{q} \beta_{i} \ln \left(\sigma_{t-i}^{2}\right)
$$


As candidate models for the distribution $z$ of the innovations we consider the skew-t distribution and the skew-GED distribution, defined by the asymmetrization construction introduced in Fernández and Steel (1998). Recall first that the densities of the standardized $t$ and GED are given by

$$
\begin{aligned}
& f(x)=\frac{\Gamma\left(\frac{\nu+1}{2}\right)}{\Gamma\left(\frac{\nu}{2}\right) \sqrt{\pi(\nu-2)}}\left(1+\frac{x^{2}}{\nu-2}\right)^{-\frac{\nu+1}{2}}, \quad \nu>2 \\
& f(x)=\frac{\kappa \exp \left(-\frac{1}{2}|x|^{\kappa}\left|\sqrt{2^{-2 / \kappa} \frac{\Gamma(1 / \kappa)}{\Gamma(3 / \kappa)}}\right|^{\kappa}\right)}{2^{1+1 / \kappa} \Gamma(1 / \kappa) \sqrt{2^{-2 / \kappa} \frac{\Gamma(1 / \kappa)}{\Gamma(3 / \kappa)}}}, \quad \kappa>0 .
\end{aligned}
$$

Given now a skew factor $\epsilon>0$, the corresponding asymmetric density is given by

$$
f_{\epsilon}(x)=\frac{2}{\epsilon+\epsilon^{-1}}\left[f(\epsilon x) \mathbf{1}_{\{x<0\}}+f\left(\epsilon^{-1} x\right) \mathbf{1}_{\{x \geq 0\}}\right] .
$$

The mean and the variance of the asymmetrized innovations $z_{\epsilon}$ can then be computed as

$$
\begin{aligned}
\mathbb{E}\left(z_{\epsilon}\right) & =M_{1}\left(\epsilon-\epsilon^{-1}\right) \\
\operatorname{Var}\left(z_{\epsilon}\right) & =\left(M_{2}-M_{1}^{2}\right)\left(\epsilon^{2}-\epsilon^{-2}\right)+2 M_{1}^{2}-M_{2},
\end{aligned}
$$

where $M_{1}=\mathbb{E}[|z|]$ and $M_{2}=\operatorname{Var}[z]$.

\section{Empirical results}

We present an empirical analysis based on a dataset of daily closing prices of S\&P500 options from 11-4-2003 to 11-4-2013. The first step of our analysis is the computation of the time series of the Interexpectile Difference $\Delta_{0.75}$. In order to have the same time horizon of the VIX, we considered options with time-to-maturities as close to 30 days as possible; the resulting options have time-to-maturities ranging from 7 to 45 days. As it is costumary in the literature, we removed a few quotations that did not satisfy standard no arbitrage relationships. In order to solve (4), we follow the procedure outlined in Bellini et al. (2018a): we first compute implied volatilities, then we interpolate them as in Shimko (1993), and then we use interpolated volatilities to compute theoretical option prices for non-quoted strikes, thus allowing the determination of implicit expectiles by numerically solving (4). The resulting Interexpectile Differences have been rescaled to a 30-day time horizon by means of the $\sqrt{\tau}$ rule already discussed in Bellini et al. (2018a).

The resulting time series of $\Delta_{0.75}$ is plotted in the lower part of Fig. 2, and compared with S\&P500 and VIX. From a purely graphical point of view, the Interexpectile Difference displays a behaviour very similar to VIX, with a peak during the financial crisis of 2008-2009, and a negative relationship with the S\&P500. These qualitative findings confirm those of Bellini et al. (2018a), that were based on a much more limited dataset of prices of options on FTSEMIB in 2008-2009.

The corresponding logreturns are reported in Fig. 3. The results of the ADF test reported in Table 1 show that nonstationarity is rejected at the $1 \%$ level for the all three series, again confirming the findings of Bellini et al. (2018a). As anticipated in Section 2, in order to remove serial autocorrelations we fitted various ARMA-GARCH and ARMA-EGARCH models (equations $(5),(6),(7))$ to the three series of logreturns, with four possible shapes of the innovations: symmetric t, symmetric GED, skew-t and skew-GED. Estimations have been performed by quasi-maximum likelihood by means of the R package rugarch (see Ghalanos et al. 2018, for the documentation).

In order to select the best fitting model and the optimal shape of innovations we have followed a two-step procedure. In the first step, for each considered shape of the innovations, the optimal 


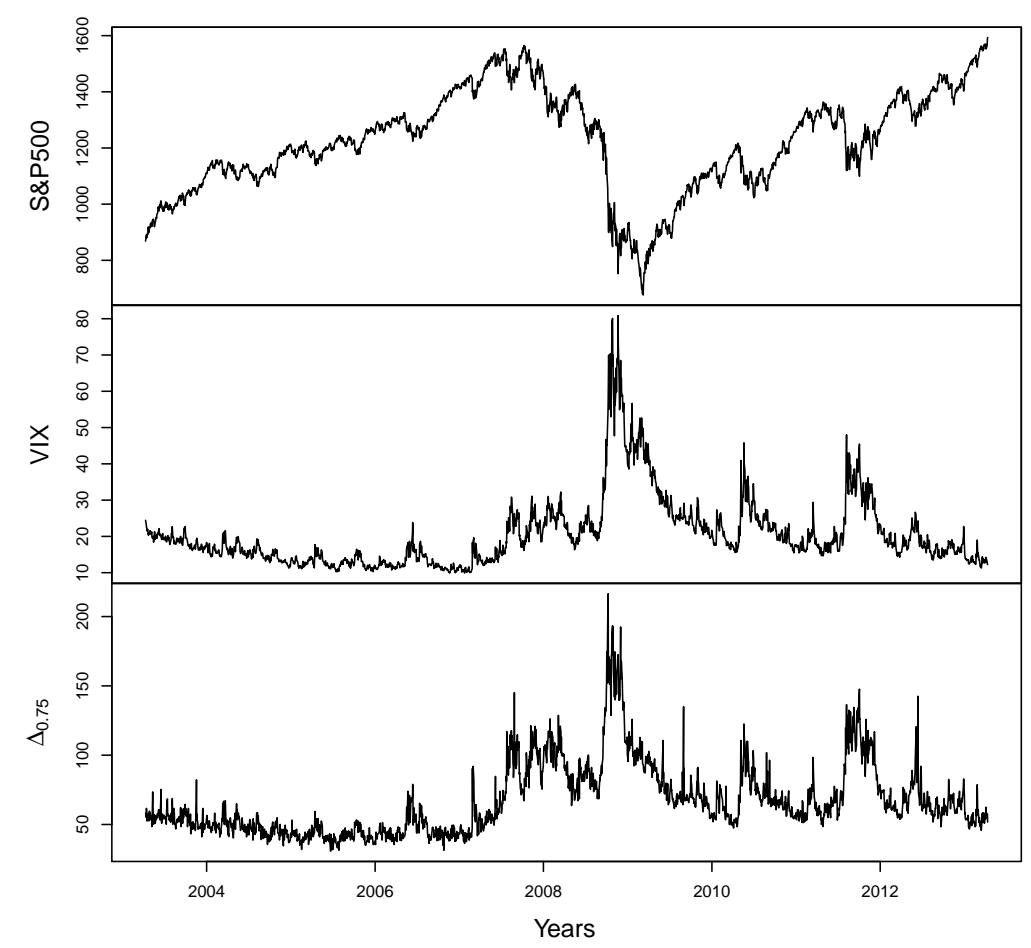

Figure 2. Time series of the levels of S\&P500, VIX and $\Delta_{0.75}$.

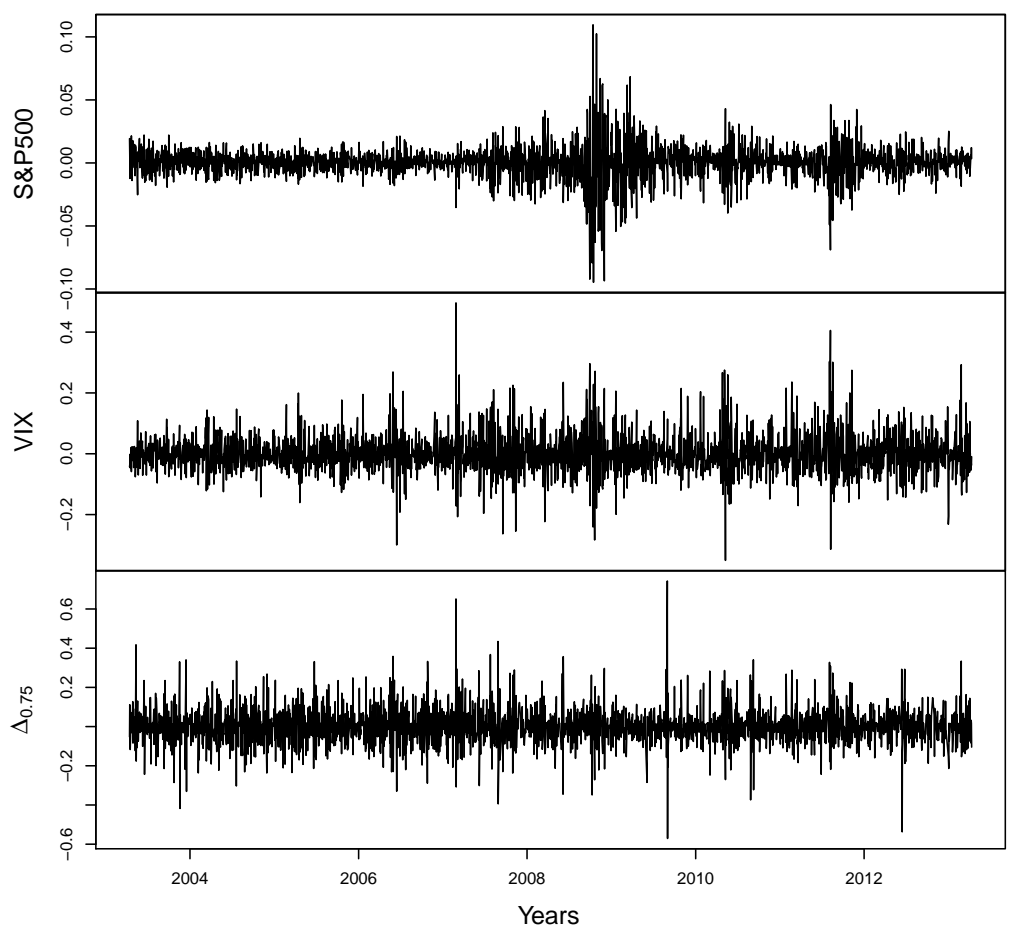

Figure 3. Time series of the logreturns of S\&P500, VIX and $\Delta_{0.75}$. 


\begin{tabular}{lccc}
\hline & S\&P500 & VIX & $\Delta_{0.75}$ \\
\hline$\hat{\gamma}$ & -1.196 & -1.211 & -1.520 \\
t-test & -40.289 & -40.640 & -48.126 \\
\hline
\end{tabular}

Table 1. Results of the ADF test for logreturns of S\&P500, VIX and $\Delta_{0.75}$. In all three cases nonstationarity is rejected at the $1 \%$ level, since the critical value is $c_{1 \%}=-2.58$.

orders $r, s$ in (5) and $p, q$ in (6) and (7) have been selected by means of the AIC criterion. In the second step, the optimal shape of the innovations has been chosen by comparing the optimal values of the AIC for the four different types of innovations under consideration.

The results are reported in Table 2. For the $\mathrm{S} \& \mathrm{P} 500$ logreturns the optimal model is $\operatorname{ARMA}(2,0)$ $\operatorname{EGARCH}(2,1)$ with skew-GED innovations with $\epsilon=0.85$ and $\kappa=1.42$, indicating negative asymmetry and exponential tails heavier than the normal, that would correspond to $\kappa=2$. For the VIX logreturns the optimal model is $\operatorname{ARMA}(2,1)$-EGARCH$(1,1)$ with skew-t innovations with $\epsilon=1.40$ and $\nu=6.34$, indicating positive asymmetry and paretian tails of moderate heavyness. Finally, for the logreturns of $\Delta_{0.75}$ the optimal model is $\operatorname{ARMA}(1,2)-\operatorname{EGARCH}(2,2)$ with skew-t innovations with $\epsilon=1.21$ and $\nu=5.01$, indicating positive asymmetry and mildly heavy tails. In all three cases, the EGARCH specification of the conditional variance seems to perform better than the GARCH specification.

\begin{tabular}{|c|c|c|c|c|}
\hline & Innovations & Best model & AIC & $\mathrm{BIC}$ \\
\hline \multirow{8}{*}{$\begin{array}{l}8 \\
8 \\
10 \\
2 \\
0 \\
0\end{array}$} & $\mathrm{t}$ & $\operatorname{ARMA}(1,1)+\operatorname{GARCH}(2,1)$ & -6.481 & -6.462 \\
\hline & GED & $\operatorname{ARMA}(1,1)+\operatorname{GARCH}(2,1)$ & -6.490 & -6.472 \\
\hline & skew-t & $\operatorname{ARMA}(1,1)+\operatorname{GARCH}(2,1)$ & -6.493 & -6.472 \\
\hline & skew-GED & $\operatorname{ARMA}(1,1)+\operatorname{GARCH}(2,1)$ & -6.503 & -6.482 \\
\hline & $\mathrm{t}$ & $\operatorname{ARMA}(2,0)+\operatorname{EGARCH}(2,1)$ & -6.516 & -6.493 \\
\hline & GED & $\operatorname{ARMA}(2,0)+\operatorname{EGARCH}(2,1)$ & -6.519 & -6.496 \\
\hline & skew-t & $\operatorname{ARMA}(1,1)+\operatorname{EGARCH}(2,1)$ & -6.532 & -6.506 \\
\hline & skew-GED & $\operatorname{ARMA}(2,0)+\operatorname{EGARCH}(2,1)$ & -6.537 & -6.511 \\
\hline \multirow{8}{*}{$\frac{x}{>}$} & $\mathrm{t}$ & $\operatorname{ARMA}(2,2)+\operatorname{GARCH}(1,1)$ & -2.866 & -2.845 \\
\hline & GED & $\operatorname{ARMA}(2,2)+\operatorname{GARCH}(1$, & -2.851 & -2.831 \\
\hline & skew- & GARCH ( & -2.910 & -2.892 \\
\hline & skew-GED & $\operatorname{ARMA}(1,1)+\operatorname{GARCH}(1,1)$ & -2.905 & -2.886 \\
\hline & $\mathrm{t}$ & $\operatorname{ARMA}(1,1)+\operatorname{EGARCH}(1,1)$ & -2.880 & -2.862 \\
\hline & GED & $\operatorname{ARMA}(2,2)+\operatorname{EGARCH}(1,1)$ & -2.866 & -2.843 \\
\hline & skew-t & $\operatorname{ARMA}(2,1)+\operatorname{EGARCH}(1,1)$ & -2.933 & -2.912 \\
\hline & skew-GED & $\operatorname{ARMA}(2,1)+\operatorname{EGARCH}(1,1)$ & -2.930 & -2.907 \\
\hline \multirow{8}{*}{$\dot{8}$} & $\mathrm{t}$ & $\operatorname{ARMA}(1,2)+\operatorname{GARCH}(2,1)$ & -2.200 & -2.179 \\
\hline & GED & $\operatorname{ARMA}(1,2)+\operatorname{GARCH}(2,1)$ & -2.185 & -2.164 \\
\hline & skew- & $\operatorname{ARMA}(1,2)+\operatorname{GARCH}(2,1$ & -2.216 & -2.193 \\
\hline & skew-GED & $\operatorname{ARMA}(1,2)+\operatorname{GARCH}(2,1)$ & -2.206 & -2.182 \\
\hline & $\mathrm{t}$ & $\operatorname{ARMA}(2,2)+\mathrm{EGARCH}(2,2)$ & -2.185 & -2.155 \\
\hline & GED & $\operatorname{ARMA}(2,2)+\operatorname{EGARCH}(2,2)$ & -2.201 & -2.171 \\
\hline & skew-1 & $\operatorname{ARMA}(1,2)+\operatorname{EGARCH}(2,2)$ & -2.219 & -2.188 \\
\hline & skew-G & $\operatorname{ARMA}(1,2)+\operatorname{EGARCH}(2,1)$ & -2.208 & -2.180 \\
\hline
\end{tabular}

Table 2. Selection of the optimal model and optimal shape of innovations for the logreturns of S\&P500, VIX and $\Delta_{0.75}$. The optimal values of AIC are boxed. The corresponding estimated parameters for the innovations are respectively $\epsilon=0.85$ and $\kappa=1.42, \epsilon=1.40$ and $\nu=6.34$, $\epsilon=1.21$ and $\nu=5.01$. 
We then compute the residuals of the three selected optimal models, and visualize their pairwise dependence structure in Fig. 4. From a purely graphical point of a view, we see a strong negative dependence between innovations of S\&P500 and VIX, a less pronounced negative dependence between innovations of S\&P500 and $\Delta_{0.75}$, and a positive dependence between the innovations of VIX and $\Delta_{0.75}$. The values of the correlation coefficients are respectively $-0.7988,-0.5238,0.6592$. As a first exploratory tool, the values of correlation between exceedances are reported in Table 3 . From these figures, we may expect radial asymmetry in all the three couples, with possible presence of asymptotic tail dependence, especially in the case of (S\&P500,VIX) and (S\&P500, $\left.\Delta_{0.75}\right)$.

\begin{tabular}{lcccccc}
\hline & \multicolumn{2}{c}{ S\&P500, VIX } & \multicolumn{2}{c}{ S\&P500, $\Delta_{0.75}$} & \multicolumn{2}{c}{ VIX, $\Delta_{0.75}$} \\
$\mathrm{q}$ & North West & South East & North West & South East & South West & North East \\
\hline 0.15 & -0.72 & -0.28 & -0.39 & 0.01 & 0.23 & 0.46 \\
0.1 & -0.73 & -0.31 & -0.37 & -0.16 & 0.16 & 0.51 \\
0.05 & -0.76 & -0.33 & -0.34 & -0.30 & 0.41 & 0.59 \\
0.025 & -0.81 & -0.17 & -0.72 & -0.16 & 0.53 & 0.70 \\
\hline
\end{tabular}

Table 3. Correlations of the exceedances displayed in Fig. 4.

The next step of our analysis is to fit the parametric families of copulas briefly reviewed in Section 2 to the empirical copulas obtained by applying the probability integral transform to the filtered innovations $\hat{z}_{i, t}$, that is by computing

$$
\hat{u}_{i, t}=\hat{F}_{i}\left(\hat{z}_{i, t}\right)
$$

where $\hat{F}_{i}$, for $i=1, \ldots, 3$, is the empirical distribution function of the innovations. Estimations have been performed by quasi-maximum likelihood by means of the $\mathrm{R}$ package vinecopula (see Schepsmeier et al. (2012)) and the best model has been selected by means of the AIC criterion in Table 4. By convention, we assign negative values of the parameters to rotated copulas.

For (S\&P500, VIX) the optimal copula is Rotated Gumbel 90 with $\theta=-2.38$, that has intermediate SE dependence and strong NW dependence. These findings are consistent with the leverage effect found in the literature, recalling that the NW quadrant corresponds to low values of the innovations of the S\&P500 and high values of the innovations of the VIX. Notice that the Rotated BB1 90, that includes the Rotated Gumbel 90 as a limiting case for $\theta \rightarrow 0$, achieves a minimally higher log-likelihood but is penalized by the AIC criterion. For $\left(\mathrm{S} \& \mathrm{P} 500, \Delta_{0.75}\right)$ the leverage effect is less pronounced, since the optimal copula is Rotated BB8 90 with $\theta=-2.58$ and $\delta=-0.88$, that has asymptotic tail independence. Notice however that the AIC of the Rotated BB8 90 copula, equal to -826.45 , is very close to the one of the Rotated Gumbel 90, that is equal to -826.41 . We will see in the subperiod analysis that the Rotated Gumbel 90 is indeed optimal in a subperiod. For (VIX, $\Delta_{0.75}$ ) the optimal copula is BB1 with $\theta=0.15$ and $\delta=1.71$, that has upper and lower strong tail dependence. The contour plots of the best-fitting copulas are reported in Fig. 5.

In order to test the stability of the results, we divided the datasets into three subperiods:

- Period 1: from 11-04-2003 to 11-04-2006

- Period 2: from 12-04-2006 to 11-04-2009

- Period 3: from 12-04-2009 to 11-04-2013,

and repeated the estimation of the ARMA-(E)GARCH models and of the parametric families of copulas on each subperiod. The selected models are reported in Table 5, while the contour plots of the optimal copulas are graphed in Fig. 6 . We see that for (S\&P500, VIX) and (S\&P500, $\left.\Delta_{0.75}\right)$ the results are quite stable: in the first case the optimal copula is always Rotated Gumbel 90, while in the second case the optimal copula is Rotated BB8 90 in the first two subperiods and Rotated Gumbel in the third subperiod. As already mentioned in connection with the analysis on the whole period, on this dataset these two models have a very similar performance. the situation is less clear 


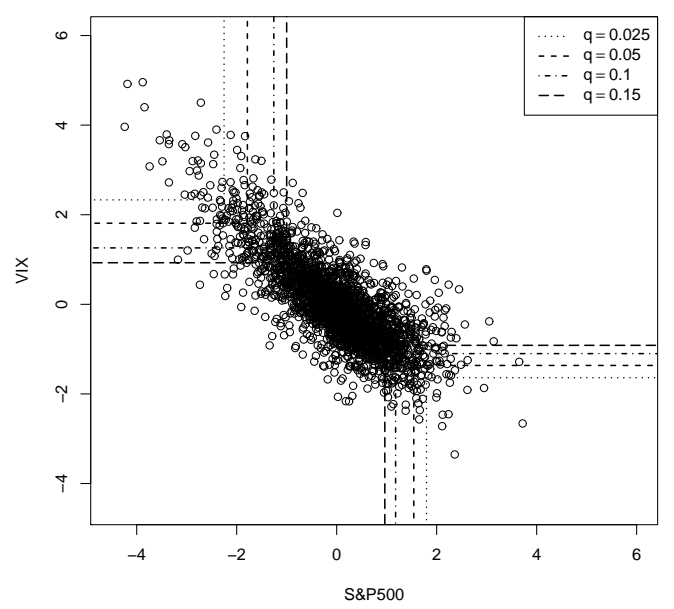

(a) S\&P500, VIX

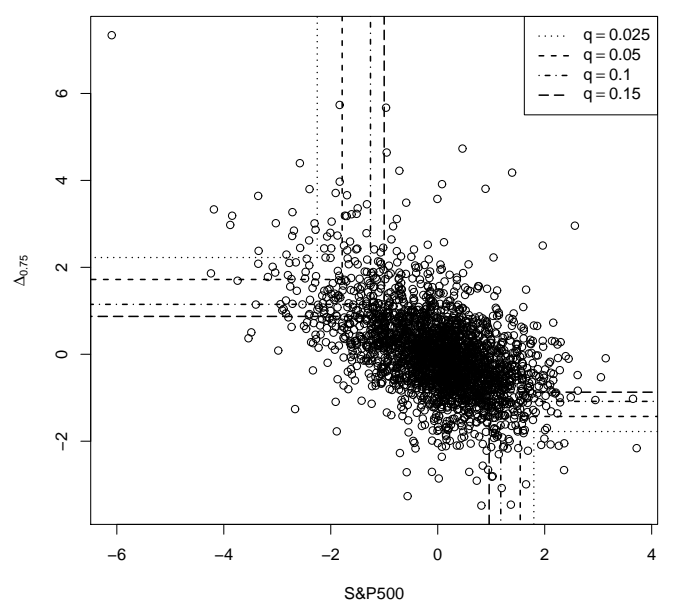

(b) $\mathrm{S} \& \mathrm{P} 500, \Delta_{0.75}$



(c) VIX, $\Delta_{0.75}$

Figure 4. Joint distribution of the residuals of the optimal models selected in Table 2. The dotted quadrants delimitate pairs of residuals that are both below (in the case of left tails) or both above (in the case of right tails) the corresponding empirical q-quantiles. 


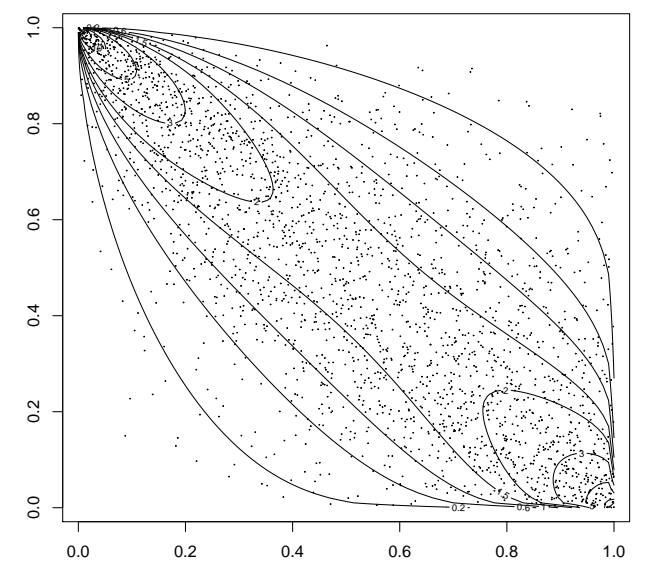

(a) S\&P500, VIX

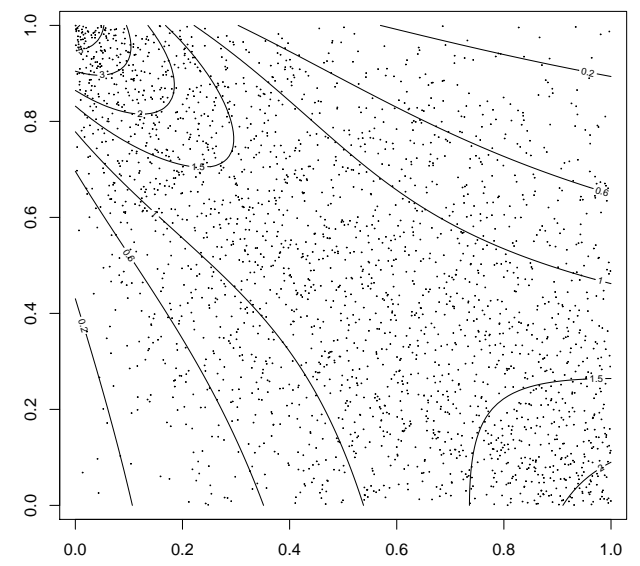

(b) S\&P $500, \Delta_{0.75}$

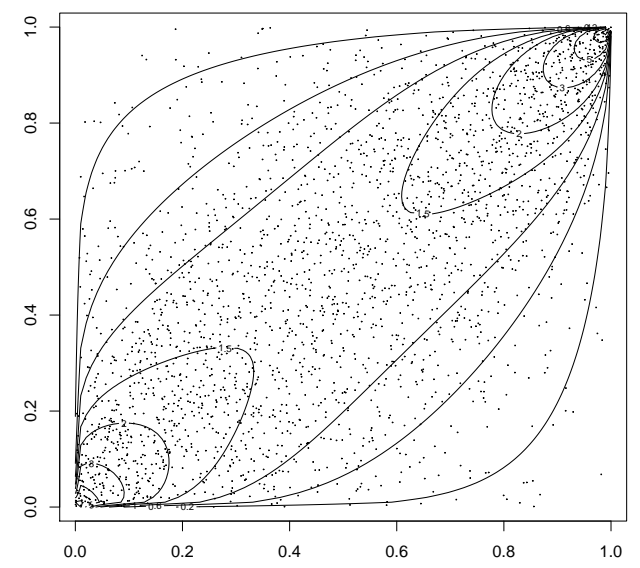

(c) VIX, $\Delta_{0.75}$

Figure 5. Empirical copulas and contour plots of the best fitting models selected in Table 4 . 


\begin{tabular}{|c|c|c|c|c|c|}
\hline Family & $\log \mathrm{L}$ & $\mathrm{AIC}$ & $\mathrm{BIC}$ & par & tau \\
\hline \multicolumn{6}{|c|}{ S\&P500, VIX } \\
\hline Gaussian & 1149.66 & -2297.32 & -2291.49 & -0.78 & -0.56 \\
\hline Frank & 1134.12 & -2266.24 & -2260.41 & -7.53 & -0.58 \\
\hline Rotated Clayton 90 & 683.09 & -1364.18 & -1358.35 & -1.36 & -0.40 \\
\hline Rotated Gumbel 90 & 1290.89 & -2579.77 & -2573.94 & -2.38 & -0.58 \\
\hline Rotated BB1 90 & 1290.91 & -2577.70 & -2566.03 & $0.00,-2.38$ & -0.58 \\
\hline Rotated BB8 90 & 1251.91 & -2499.82 & -2488.16 & $-4.13,-0.93$ & -0.57 \\
\hline Rotated Clayton 270 & 1212.79 & -2423.58 & -2417.74 & -2.24 & -0.53 \\
\hline Rotated Gumbel 270 & 970.99 & -1939.99 & -1934.16 & -2.1 & -0.52 \\
\hline Rotated BB1 270 & 1276.98 & -2549.95 & -2538.29 & $-1.38,-1.37$ & -0.57 \\
\hline Rotated BB8 270 & 1005.29 & -2006.57 & -1994.91 & $-6,-0.7$ & -0.53 \\
\hline \multicolumn{6}{|c|}{$\mathrm{S} \& \mathrm{P} 500, \Delta_{0.75}$} \\
\hline Gaussian & 382.85 & -763.71 & -757.88 & -0.51 & -0.34 \\
\hline Frank & 378.48 & -754.97 & -749.14 & -3.61 & -0.36 \\
\hline Rotated Clayton 90 & 215.19 & -428.38 & -422.55 & -0.61 & -0.23 \\
\hline Rotated Gumbel 90 & 414.21 & -826.41 & -820.58 & -1.51 & -0.34 \\
\hline Rotated BB1 90 & 414.37 & -824.74 & -813.08 & $-0.02,-1.5$ & -0.34 \\
\hline Rotated BB8 90 & 415.23 & -826.45 & -814.79 & $-2.58,-0.88$ & -0.35 \\
\hline Rotated Clayton 270 & 389.28 & -776.55 & -770.72 & -0.86 & -0.30 \\
\hline Rotated Gumbel 270 & 302.19 & -602.38 & -596.55 & -1.44 & -0.31 \\
\hline Rotated BB1 270 & 411.55 & -819.11 & -807.45 & $-0.62,-1.15$ & -0.33 \\
\hline Rotated BB8 270 & 353.55 & -703.10 & -691.44 & $-6,-0.47$ & -0.34 \\
\hline \multicolumn{6}{|c|}{ VIX, $\Delta_{0.75}$} \\
\hline Gaussian & 700.72 & -1399.44 & -1393.61 & 0.65 & 0.45 \\
\hline Clayton & 466.49 & -930.98 & -925.15 & 1.01 & 0.34 \\
\hline Gumbel & 727.59 & -1453.18 & -1447.35 & 1.82 & 0.45 \\
\hline Frank & 706.41 & -1410.82 & -1404.99 & 5.34 & 0.48 \\
\hline BB1 & 736.00 & \begin{tabular}{|l|}
-1468.01 \\
\end{tabular} & -1456.35 & $0.15,1.71$ & 0.46 \\
\hline BB8 & 728.28 & -1452.56 & -1440.90 & $4.93,0.72$ & 0.47 \\
\hline Survival Clayton & 639.22 & -1276.44 & -1270.61 & 1.27 & 0.39 \\
\hline Survival Gumbel & 620.91 & -1239.81 & -1233.98 & 1.74 & 0.43 \\
\hline Survival BB1 & 729.55 & -1455.11 & -1443.44 & $0.64,1.38$ & 0.45 \\
\hline Survival BB8 & 664.01 & -1324.02 & -1312.36 & $6,0.6$ & 0.45 \\
\hline
\end{tabular}

Table 4. Selection of best-fitting copulas by means of the AIC criterion for the three couples (S\&P500, VIX), (S\&P500, $\left.\Delta_{0.75}\right)$ and (VIX, $\left.\Delta_{0.75}\right)$. The corresponding parameters and Kendall's $\tau$ are reported in the last two columns. By convention, a negative sign is attributed to the parameters of rotated copulas.

for the couple (VIX, $\Delta_{0.75}$ ), where the optimal models in the three subperiods are respctively BB8, Frank and BB1, so tail dependence appears only in the third subperiod.

\section{Conclusions}

The first important finding of the paper is that Interexpectile Differences seem to be a viable alternative to the VIX index for measuring implied variability. Indeed, the two time series displays a very similar behaviour on the whole considered 2003-2013 period. Both implied variability indices display a similar leverage effect, that is a negative dependence between variations in the VIX or in the Interexpectile Difference and contemporary variations in the SP\&500. A more refined 


\begin{tabular}{|c|c|c|c|}
\hline & Period 1 & Period 2 & Period 3 \\
\hline \multirow[t]{2}{*}{ S\&P500 } & $\operatorname{ARMA}(1,2)+\mathrm{EGARCH}(2,1)$ & $\operatorname{ARMA}(0,2)+\operatorname{EGARCH}(2,1)$ & $\operatorname{ARMA}(0,1)+\operatorname{EGARCH}(2,1)$ \\
\hline & skew-GED $(\epsilon=0.89, \kappa=1.90)$ & skew-GED $(\epsilon=0.84, \kappa=1.29)$ & skew-GED $(\epsilon=0.86, \kappa=1.39)$ \\
\hline \multirow[t]{2}{*}{ VIX } & $\operatorname{ARMA}(2,1)+\mathrm{EGARCH}(1,1)$ & $\operatorname{ARMA}(1,1)+\operatorname{EGARCH}(1,1)$ & $\operatorname{ARMA}(1,2)+\operatorname{EGARCH}(1,1)$ \\
\hline & skew-t $(\epsilon=1.37, \nu=12.26)$ & skew-GED $(\epsilon=1.49, \kappa=1.30)$ & skew-t $(\epsilon=1.36, \nu=3.77)$ \\
\hline \multirow[t]{2}{*}{$\Delta_{0.75}$} & $\operatorname{ARMA}(1,2)+\operatorname{GARCH}(1,1)$ & $\operatorname{ARMA}(2,2)+\operatorname{GARCH}(2,1)$ & $\operatorname{ARMA}(2,1)+\operatorname{EGARCH}(1,1)$ \\
\hline & $\mathrm{t}(\nu=7.00)$ & skew-GED $(\epsilon=1.25, \kappa=1.25)$ & skew-t $(\epsilon=1.23, \nu=4.28)$ \\
\hline \multirow[t]{2}{*}{ S\&P500, VIX } & Rotated Gumbel 90 & Rotated Gumbel 90 & Rotated Gumbel 90 \\
\hline & $\theta=-2.03, \tau=-0.51$ & $\theta=-2.55, \tau=-0.61$ & $\theta=-2.4, \tau=-0.57$ \\
\hline \multirow[t]{2}{*}{$\mathrm{S} \& \mathrm{P} 500, \Delta_{0.75}$} & Rotated BB8 90 & Rotated BB8 90 & Rotated Gumbel 90 \\
\hline & $\theta_{1}=-1.70, \theta_{2}=-0.97, \tau=-0.25$ & $\theta_{1}=-3.13, \theta_{2}=-0.78, \tau=-0.35$ & $\theta=-1.65, \tau=-0.39$ \\
\hline \multirow[t]{2}{*}{ VIX, $\Delta_{0.75}$} & BB8 & Frank & BB1 \\
\hline & $\theta_{1}=3.86, \theta_{2}=0.73, \tau=0.39$ & $\theta=5.34, \tau=0.48$ & $\theta_{1}=0.13, \theta_{2}=1.80, \tau=0.50$ \\
\hline
\end{tabular}

Table 5. Results of the subperiod analysis. In the upper part we report the best fitting model selected by means of the AIC criterion as in Table 2 and the corresponding optimal parameters. In the lower part we report the best fitting copulas selected by means of the AIC criterion as in Table 4, the corresponding parameters and the value of Kendall's $\tau$. By convention, a negative sign is attributed to the parameters of rotated copulas.

analysis based on estimating copulas on the residuals of ARMA-(E)GARCH models shows that the dependence structure is typically not symmetric and is optimally captured by a Rotated 90 copula, which seems also relatively stable among various subperiods. The situation is less clear for the positive dependence structure between VIX and Interexpectile Differences, which is optimally captured by a BB1 copula, that does not show asymptotic tail dependence. 


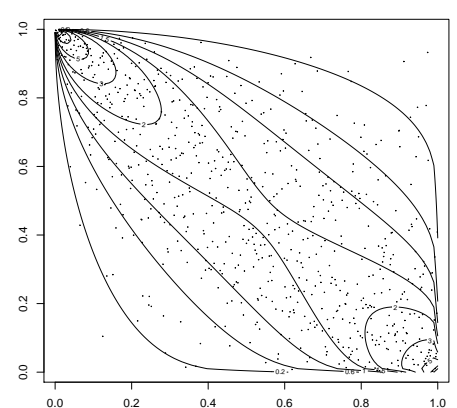

(a) S\&P500, VIX Period 1.

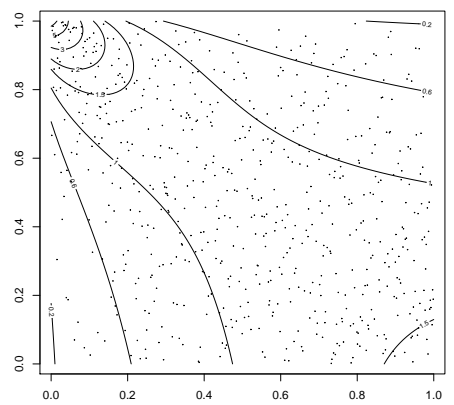

(d) S\&P500, $\Delta_{0.75}$ Period 1.

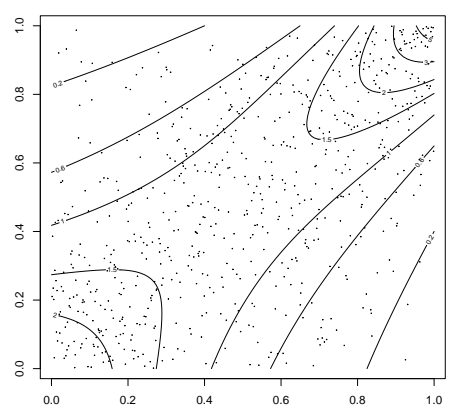

(g) VIX, $\Delta_{0.75}$ Period 1.

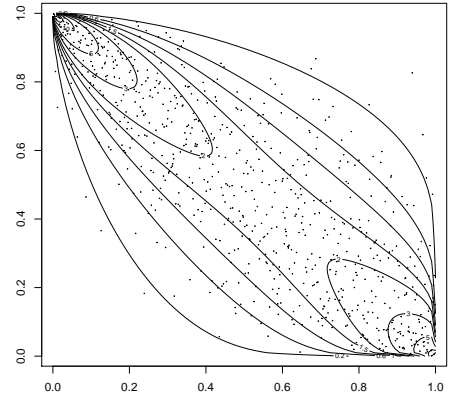

(b) S\&P500, VIX Period 2

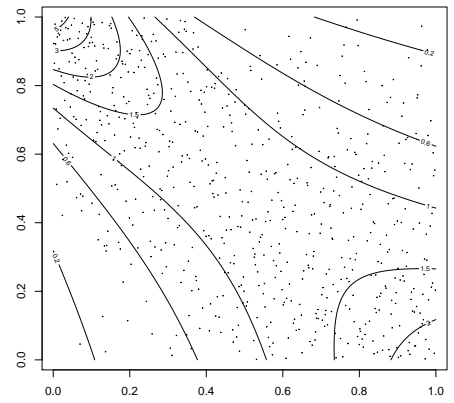

(e) S\&P500, $\Delta_{0.75}$ Period 2 .

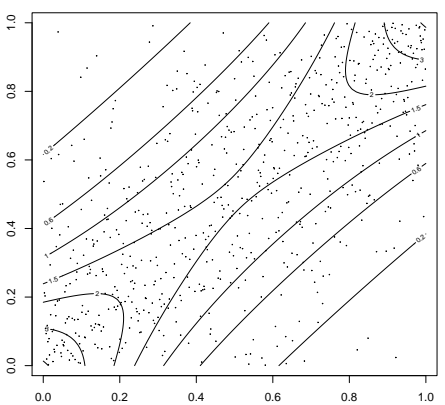

(h) VIX, $\Delta_{0.75}$ Period 2 .

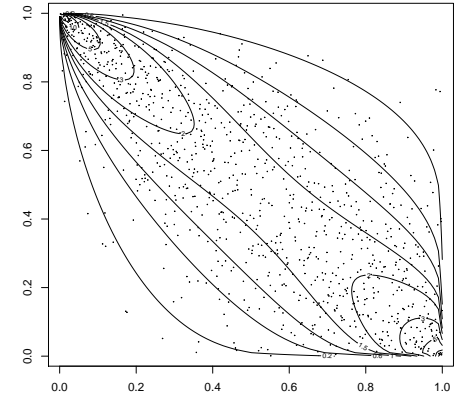

(c) S\&P500, VIX Period 3.



(f) $\mathrm{S} \& \mathrm{P} 500, \Delta_{0.75}$ Period 3.

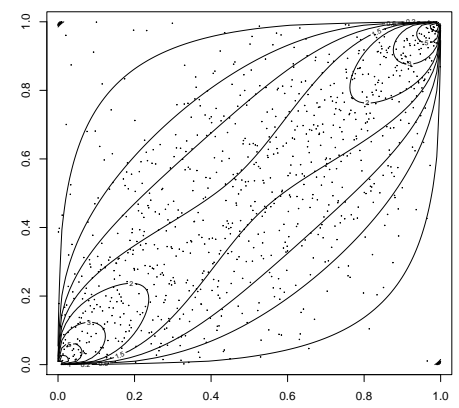

(i) VIX, $\Delta_{0.75}$ Period 3.

Figure 6. Results of the subperiod analysis. Empirical copulas and contour plots of the best fitting models reported in Table 5 . 


\section{References}

Ammann, M. and Süss, S., Asymmetric dependence patterns in financial time series. The European Journal of Finance, 2009, 15, 703-719.

Banerjee, P.S., Doran, J. and Peterson, D., Implied volatility and future portfolio returns. Journal of Banking and Finance, 2007, 31, 3183-3199.

Bellini, F., Klar, B., Müller, A. and Rosazza Gianin, E., Generalized quantiles as risk measures. Insurance: Mathematics and Economics, 2014, 54, 41-48.

Bellini, F., Mercuri, L. and Rroji, E., Implicit expectiles and measures of implied volatility. Quantitative Finance, 2018a, pp. 1-14.

Bellini, F., Klar, B. and Müller, A., Expectiles, Omega Ratios and Stochastic Ordering. Methodology and Computing in Applied Probability, 2018b, 20, 855-873.

CBOE, The CBOE Volatility Index: VIX. CBOE White Paper, 2009.

Demeterfi, K., Derman, E., Kamal, K. and Zou, J., More Than You Ever Wanted To Know About Volatility Swaps. Quantitative Strategies Research Notes Goldman Sachs \& Co, 1999.

Fernández, C. and Steel, M.F.J., On Bayesian Modeling of Fat Tails and Skewness. Journal of the American Statistical Association, 1998, 93, 359-371.

Fink, H., Klimova, Y., Czado, C. and Stöber, J., Regime switching vine copula models for global equity and volatility indices. Econometrics, 2017, 5, 3.

Ghalanos, A., Ghalanos, M.A. and Rcpp, L., Package 'rugarch'. , 2018.

Joe, H., Dependence modeling with copulas, 2014, Chapman and Hall/CRC.

Mai, J. and Scherer, M., Financial engineering with copulas explained, 2014, Springer.

Nelsen, R.B., An Introduction to Copulas (Springer Series in Statistics), 2006 (Springer-Verlag: Berlin, Heidelberg).

Newey, W. and Powell, J., Asymmetric least squares estimation and testing. Econometrica, 1987, 55, 819847.

Patton, A.J., Modelling asymmetric exchange rate dependence. International economic review, 2006, 47, $527-556$.

Peng, Y. and Ng, W.L., Analysing financial contagion and asymmetric market dependence with volatility indices via copulas. Annals of Finance, 2012a, 8, 49-74.

Peng, Y. and Ng, W.L., Extreme Spillover Effects of Volatility Indices. Journal of Economic Research, 2012b, $17,1-17$.

Rubbaniy, G., Asmerom, R., Rizvi, S.K.A. and Naqvi, B., Do fear indices help predict stock returns?. Quantitative Finance, 2014, 14, 831-847.

Schepsmeier, U., Stoeber, J., Brechmann, E.C., Graeler, B., Nagler, T., Erhardt, T. et al., VineCopula: Statistical inference of vine copulas. $R$ package version, 2012, 1.

Shimko, D., Bounds of probability. Risk, 1993, 6, 33-37. 